\title{
Transitions in hookah (Waterpipe) smoking by U.S. sexual minority adults between 2013 and 2015: the population assessment of tobacco and health study wave 1 and
} wave 2

Mary Rezk-Hanna ${ }^{1 *}$, lan W. Holloway², Joy Toyama', Umme Shefa Warda', Lorree Catherine Berteau', Mary-Lynn Brecht ${ }^{1}$ and Linda Sarna ${ }^{1}$

\begin{abstract}
Background: Tobacco smoking using a hookah (i.e., waterpipe) is a global epidemic. While evidence suggests that sexual minorities (SM) have higher odds of hookah use compared to heterosexuals, little is known about their hookah use patterns and transitions. We sought to examine transitions between hookah smoking and use of other tobacco and electronic (e-) products among SM adults aged 18 years of age and older versus their heterosexual counterparts.

Methods: We analyzed nationally representative data of ever and current hookah smokers from Wave 1 (20132014; ever use $n=1014$ SM and $n=9462$ heterosexuals; current use $n=144$ SM and $n=910$ heterosexuals) and Wave 2 (2014-2015; ever use $n=901$ SM and $n=8049$ heterosexuals; current use $n=117$ SM and $n=602$ heterosexuals) of the Population Assessment of Tobacco and Health Study. Comparisons between groups and gender subgroups within SM identity groups were determined with Rao-Scott chi-square tests and multivariable survey-weighted multinomial logistic regression models were estimated for transition patterns and initiation of electronic product use in Wave 2.
\end{abstract}

Results: Ever and current hookah smoking among SM adults (ever use Wave 1: 29\% and Wave 2: 31\%; current use Wave 1: $4 \%$ and Wave 2: 3\%) was higher than heterosexuals (ever use Wave 1: 16\% and Wave 2: 16\%; current use Wave 1: $1 \%$ and Wave 2: $1 \%$; both $p<0.0001$ ). Among SM adults who reported hookah use at Wave 1, 46\% quit hookah use at Wave 2; 39\% continued hookah use and did not transition to other products while 36\% of heterosexual adults quit hookah use at Wave 2 and 36\% continued hookah use and did not transition to other products. Compared with heterosexuals, SM adults reported higher use of hookah plus e-products (Wave 2 usage increased by 65 and 83\%, respectively).

(Continued on next page)

\footnotetext{
* Correspondence: mrezk@ucla.edu

'School of Nursing, University of California, Los Angeles, 700 Tiverton Ave, 4-254 Factor Building, Los Angeles, CA 90095, USA

Full list of author information is available at the end of the article
}

(c) The Author(s). 2021 Open Access This article is licensed under a Creative Commons Attribution 4.0 International License, which permits use, sharing, adaptation, distribution and reproduction in any medium or format, as long as you give appropriate credit to the original author(s) and the source, provide a link to the Creative Commons licence, and indicate if changes were made. The images or other third party material in this article are included in the article's Creative Commons. licence, unless indicated otherwise in a credit line to the material. If material is not included in the article's Creative Commons licence and your intended use is not permitted by statutory regulation or exceeds the permitted use, you will need to obtain permission directly from the copyright holder. To view a copy of this licence, visit http://creativecommons.org/licenses/by/4.0/. The Creative Commons Public Domain Dedication waiver (http://creativecommons.org/publicdomain/zero/1.0/) applies to the data made available in this article, unless otherwise stated in a credit line to the data. 
(Continued from previous page)

Conclusions: Compared to heterosexuals, in addition to higher rates of hookah smoking, higher percentages of SM adults transitioned to hookah plus e-product use between 2013 and 2015. Results have implications for stronger efforts to increase awareness of the harmful effects of hookah as well as vaping, specifically tailored among SM communities.

Keywords: Hookah, Waterpipe, Sexual minority, Tobacco, Vaping

\section{Background}

Tobacco smoking using a hookah (i.e., waterpipe) is a global epidemic [1]. Contributing to hookah's popularity is the unsubstantiated belief that smoke is detoxified as it passes through water, rendering hookah as a safer tobacco alternative [2-4]. Tobacco and alternative tobacco products are disproportionately being used by sexual minority (SM) adults (i.e., lesbian, gay and bisexual individuals) [58]. According to Wave 1 Population Assessment of Tobacco and Health (PATH) Study (2013-2014), 39.8\% of lesbian/gay adults and $45.7 \%$ of bisexual adults reported current tobacco use, compared to $27.3 \%$ of heterosexual individuals [9]. Lesbian and bisexual women (18 years of age and older) had higher odds of experimental and regular use of hookah compared to heterosexual women [10]. Similarly, gay identified men $\geq 25$ years of age had higher odds of experimental hookah use.

While prevalence rates provide useful information about hookah use among SM adults, to date, virtually nothing is known about how SM hookah smokers have quit or transitioned over time to other tobacco products, including electronic nicotine delivery systems such as ecigarettes. Understanding changes in tobacco use behavior over time is imperative for providing insight into the net population health impact of tobacco use as well as how to support quit efforts. This is specifically important given the known tobacco-use disparities among SM individuals. Indeed, common smoking risk factors, including stress and depression-experienced at higher rates among SM adults compared to heterosexual adultshave been shown to play a vital role in etiologies of tobacco-related disparities and may make quitting more difficult [11]. Additionally, the tobacco industry has aggressively targeted sexual and racial/ethnic minorities through specifically designed marketing campaigns, community outreach and promotions [12]. Research shows these populations face higher risk of being exposed to online tobacco marketing and are more likely to interact with tobacco-related messages on social media compared to their heterosexual counterparts [1316]. In particular, SM women have reported more exposure to tobacco industry marketing than heterosexual women [17].

Among the general population, recent longitudinal nationally representative data from PATH study show that while the overall prevalence of tobacco product use decreased (from 28 to 26\%) from Wave 1 (2013-2014) to Wave 2 (2014-2015), over half of U.S. adult tobacco users transitioned in product use or combination of products used [18]. Among Wave 1 tobacco users, 72\% of young adults (18-24 years of age) transitioned to use other products, including non-combustible and electronic nicotine devices; $20.7 \%$ discontinued use completely; and $45.9 \%$ of older adults ( $\geq 25$ years of age) transitioned to other products, with $12.5 \%$ discontinuing use completely.

Transitions in hookah use to other tobacco products or quitting all together among SM adults remains unknown. Accordingly, using Wave 1 (2013-2014) and Wave 2 (2014-2015) survey data from the PATH Study, the objective of this study was to characterize transitions between hookah smoking and use of other tobacco products, including cigarettes, cigars, cigarillos, smokeless tobacco, pipe tobacco, snus pouches, dissolvable tobacco and electronic (e-) nicotine products, among SM adults aged 18 years of age and older versus their heterosexual counterparts.

\section{Methods}

\section{Study design}

We used data for adults 18 years and older from Wave 1 (September 12, 2013, to December 14, 2014) and Wave 2 (October 23, 2014, to October 30, 2015) of the PATH Study, a nationally representative, longitudinal cohort study of non-institutionalized adult and youth residents of the U.S. ages 12 and older. The PATH Study was designed to collect data on use patterns, risk perceptions, attitudes and health outcomes associated with tobacco and alternative tobacco products [19]. The PATH study design oversampled adult tobacco users, young adults (aged 18-24) and African-American adults, relative to population proportions. Weighting procedures adjusted for oversampling and allowed for representation of noninstitutionalized, civilian US population. A detailed overview of the PATH study design and methods are reported elsewhere $[19,20]$. The PATH study was approved by Westat's Institutional Review Board, and the United States Office of Management and Budget approved the data collection. Secondary data analysis of 
the PATH Study Files was approved by the University of California, Los Angeles Institutional Review Board.

\section{Measures \\ Socio-demographic characteristics}

Data on sex (male vs. female) and sexual orientation (lesbian, gay, bisexual or something else vs. heterosexual), was collected during each wave. Sexual orientation was self-identified by asking respondents to answer the following question: "Do you think of yourself as: (a) "Lesbian or gay", (b) "Straight, that is, not lesbian or gay", (c) "Bisexual", or (d) "Something else". Participants who reported "something else" were probed to provide additional clarifying information (i.e., identifying with other labels such a queer, transgender, in the process of figuring out their sexual orientation, not having a sexuality, not using such labels, or something else). For the purpose of this paper, sexual minorities were defined as lesbian or gay, bisexual or something else, while heterosexuals were defined as straight. Additional demographic data included age, race/ ethnicity, education level, marital status, health insurance status, and annual household income. Age in years was classified as 18-24, 25-34, $35-44,45-54$, and $\geq 55$. Race/ethnicity was classified as white non-Hispanic, black non-Hispanic, other nonHispanic, and Hispanic. Education level was categorized by college or no college. Marital status was categorized as married and non-married. Non-married included widowed, divorced, separated or never married. Annual household income was categorized into income categories: < \$25,000, \$25,000-49,999, \$50,000-99,999 and $>\$ 100,000$.

\section{Hookah and tobacco use patterns and transitions}

Ever hookah use was defined as lifetime use. Current hookah use was defined as currently smoking hookah every day or some days (in past 30 days). Study participants were not mutually exclusive to hookah use; that is, some participants who used hookah may also have used other tobacco products including cigarettes, cigar, traditional cigars, filtered cigars, cigarillos, electronic devices, smokeless tobacco (i.e., loose snus, moist snuff, dip, spit, or chewing tobacco), pipe tobacco, snus pouches, or dissolvable tobacco. Categories of single- and multipleproduct use for purposes of this paper are described in more detail in the next section.

Among the subset of respondents who reported current hookah only use (no other tobacco products) at Wave 1, four types of transitions to Wave 2 tobacco products were examined: (a) No transition in hookah use (i.e., hookah use at Wave 2 as used at Wave 1); (b) Continued hookah and transitioned to other tobacco product(s) (i.e., hookah plus other tobacco product(s) use at Wave 2); (c) Quit hookah and transitioned to other tobacco product(s) (i.e., no hookah use but other tobacco product(s) use at Wave 2); and (d) Quit all tobacco use (i.e., no use of hookah or any tobacco product at Wave 2).

\section{Co-use of tobacco, alternative tobacco products and nicotine delivery systems}

To assess for co-use of other tobacco and e-nicotine products, single, dual and poly hookah use were examined using five broad product categories: (a) hookah; (b) cigarettes; (c) e-products (i.e., e-cigarettes, e-hookah (Wave 2 only), e-pipe (Wave 2 only)); (d) smokeless tobacco (i.e., snus, moist snuff, dip, spit, chewing tobacco or dissolvable tobacco); and other combustibles (i.e., traditional cigars, filtered cigars, cigarillos, pipe tobacco). Those who used hookah only were classified as single hookah users. Those who concurrently used hookah plus one other product category were classified as dual hookah users, and those who concurrently used hookah plus 2 or more other product categories were classified as poly hookah users. To emphasize the transition of inclusion of e-products, the following three categories were examined: (a) hookah; (b) hookah plus e-products; and (c) hookah plus other tobacco products, including cigarettes, smokeless tobacco and other combustibles.

\section{Statistical analyses}

Weighted percentages and means along with their corresponding 95\% confidence intervals (CI) were calculated using SAS 9.4. Analyses were estimated using the balanced repeated replication (BRR) method with a Fay's variant to utilize the replicate weights. Comparisons between groups (SM vs. heterosexuals) or between gender subgroups within SM identity groups on demographic variables were determined with Rao-Scott chi-square tests. Supplemental multivariable survey-weighted logistic regression analyses further explored sociodemographic characteristics associated with ever and current hookah use at Wave 1 and Wave 2. Age, gender, sexual orientation, race/ethnicity, education, income, insurance, as well as two-way interactions of sexual orientation with the other predictors, were included in the models.

Additional multivariable survey-weighted multinomial logistic regression models were estimated for selected transition patterns. For these analyses, because of sparse data coverage, age categories were collapsed to $18-24$ and 25 years of age or older. Models were developed in a stepwise manner adding one main effect at a time (same predictors as listed for logistic regression), retaining those with $p<0.40$, and similarly for relevant interactions of sexual orientation with the included effects, until estimation failed. Data would not support estimation of models with all possible transition categories for use status and multi-product use; thus more general 
categories were defined. The first analysis considered transitions from hookah-only use in Wave 1 to use status in Wave 2, specifically the following patterns: continued use of hookah only, use of other tobacco products in addition to hookah, use of other tobacco products but no hookah use, and no use of any tobacco product. Raw sample size was 322; only main effects of age, gender, and sexual orientation could be included in this model for estimation to be attained. The second transition analysis considered specifically the initiation of electronic product use in Wave 2 for Wave 1 current hookah users. Transition patterns included 1) consistency of product use from Wave 1 to Wave 2 (i.e., continuous hookahonly, continuous hookah plus e-products [with or without other tobacco products], or continuous hookah plus other tobacco products [no e-products]); 2) initiation of e-product use at Wave 2 in addition to continued hookah use; 3) continued hookah use along with any other change in use of other tobacco products; 4) cessation of hookah use. Raw sample size was 743; only the age-bysexual orientation interaction could be included in the model along with the main effects for age, gender, sexual orientation, race/ethnicity, and health insurance status.

\section{Results}

Prevalence, socio-demographic and other characteristics

Table 1 presents the prevalence of hookah use by selfreported sexual identity. Overall, SM ever hookah use (Wave 1: 29.3\%; Wave 2: 30.9\%; weighted) was higher than heterosexual ever hookah use (Wave 1: 16.0\%; Wave 2: $15.8 \% ; p<0.05$ ). Similarly, SM current hookah use (Wave 1: 3.6\%; Wave 2: 3.2\%) was higher than heterosexual current hookah use (Wave 1: 1.3\%; Wave 2: 1.0\%; $\mathrm{p}<0.05$ ). Among SM adults, females reported a higher prevalence of both ever (30.2, 31.5\%) and current (4.2, 3.5\%) hookah use than males (ever use: 28.0, 32.4\%; current use: 2.6, 2.7\%) in Wave 1 and Wave 2, respectively. Among heterosexual adults the opposite pattern was observed, with males reporting higher prevalence of ever $(19.4,19.1 \%)$ and current $(1.7,1.4 \%)$ hookah use than did females (ever use: 12.8, 12.7\%; current use: $0.8,0.7 \%)$ for Wave 1 and Wave 2 , respectively $(p<$ 0.05).

Table 2 presents the demographic characteristics of each self-reported sexual identity group in Wave 1 and Wave 2. Both SM and heterosexual participants who used hookah were predominantly aged $18-24$ or $25-34$ years old. Compared with heterosexual respondents, SM ever and current hookah users were more likely to be female (ever use: in Wave $162.9 \%$ female vs. $37.0 \%$ male, in Wave $260.0 \%$ vs. $40.0 \%$; current use: in Wave 1 $71.8 \%$ vs. $28.2 \%$, in Wave $265.4 \%$ vs. $34.6 \%$ ). Conversely, the sample of heterosexuals who used hookah was predominantly male (i.e., ever use in Wave $159.3 \%$ male vs. $40.7 \%$ female) $(p<0.05)$.

In multivariable models, relatively few of the included sociodemographic characteristics (or their interactions with sexual minority status) were consistently statistically significantly associated with ever or current hookah use (Supplemental Table 1). A consistently statistically significant effect across both waves for ever and current use was the gender by sexual minority interaction, with greater likelihood of sexual minority hookah users being female vs. male than among heterosexual hookah users (supporting the simpler comparisons described above). Education was a consistent statistically significant effect, where those with some college were likely to report ever or current use compared to those with no college. In 3 of the 4 models, where the age main effect can be interpreted (Waves 1 and 2 ever use and Wave 1 current use), the older age groups were less likely than the 1824 year old group to report ever use or current use of hookah, with decreasing likelihood as age increases.

\section{Hookah smoking transitions from wave 1 to wave 2}

Figure 1 depicts transition patterns by gender and age breakdown among SM adult Wave 1 current hookahonly smokers versus their heterosexual counterparts. Among SM current hookah smokers at Wave 1 who did not use other tobacco products (referred to here as "current hookah-only smokers"), $38.8 \%$ continued to

Table 1 Prevalence of Hookah Use Stratified by Self-Reported Sexual Identity and Gender

\begin{tabular}{|c|c|c|c|c|}
\hline \multirow{3}{*}{ 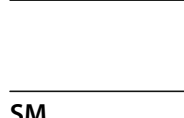 } & \multicolumn{2}{|l|}{ Ever Use } & \multicolumn{2}{|l|}{ Current Use } \\
\hline & Wave 1 Total $(n=10,604)$ & Wave 2 Total $(n=9021)$ & Wave 1 Total $(n=1058)$ & Wave 2 Total $(n=723)$ \\
\hline & $1014(29.31)^{\mathrm{a}}$ & $901(30.91)^{a}$ & $144(3.58)^{\mathrm{a}}$ & $117(3.15)^{\mathrm{a}}$ \\
\hline Male & $322(27.96)^{b}$ & $291\left(30.37^{b}\right.$ & $39(2.6)^{\mathrm{b}}$ & $33(2.67)^{b}$ \\
\hline Female & $691(30.23)$ & $609(31.5)$ & $105(4.21)$ & $84(3.5)$ \\
\hline Heterosexual & $9462(16.01)$ & 8049 (15.79) & $910(1.26)$ & $602(1.01)$ \\
\hline Male & $5427(19.44)$ & $4462(19.12)$ & $593(1.73)$ & $374(1.35)$ \\
\hline Female & 4033 (12.75) & 3584 (12.66) & $317(0.82)$ & $228(0.69)$ \\
\hline
\end{tabular}

Data represent unweighted numbers (weighted \%). Total unweighted numbers for PATH; Wave $1=32,548$; Wave $2=28,362$

${ }^{a} P<0.0001$ comparing weighted rates for SM to heterosexual for ever use (vs. never use) within wave and for current use (vs. no current use) within wave

${ }^{\mathrm{b}} \mathrm{P}<0.0001$ for $\mathrm{SM}$ comparing weighted rates for males vs. females for ever use (vs. never use) and for current use vs. no current use within wave; and similarly

for heterosexuals 
Table 2 Demographic Characteristics of Survey Participants Who Smoke Hookah*

\begin{tabular}{|c|c|c|c|c|c|c|c|c|}
\hline \multirow[t]{3}{*}{ Characteristic } & \multicolumn{4}{|l|}{ Ever Use } & \multicolumn{4}{|l|}{ Current Use } \\
\hline & \multicolumn{2}{|c|}{ Sexual Minority Adults } & \multicolumn{2}{|c|}{ Heterosexual Adults } & \multicolumn{2}{|c|}{ Sexual Minority Adults } & \multicolumn{2}{|c|}{ Heterosexual Adults } \\
\hline & Wave 1 & Wave 2 & Wave 1 & Wave 2 & Wave 1 & Wave 2 & Wave 1 & Wave 2 \\
\hline \multicolumn{9}{|l|}{ Age } \\
\hline $18-24 \mathrm{Yr}$ & $\begin{array}{l}43.11(39.5- \\
46.72)\end{array}$ & $\begin{array}{l}42.31(38.74- \\
45.88)\end{array}$ & $\begin{array}{l}34.64(33.37- \\
35.91)\end{array}$ & $\begin{array}{l}34.36(32.98- \\
35.73)\end{array}$ & $\begin{array}{l}67.41(57.85- \\
76.97)\end{array}$ & $\begin{array}{l}69.25(59.04- \\
79.47)\end{array}$ & $\begin{array}{l}64.89(60.96- \\
68.82)\end{array}$ & $\begin{array}{l}63.02(57.66- \\
68.37)\end{array}$ \\
\hline $25-34 \mathrm{Yr}$ & $\begin{array}{l}32.71(29.17- \\
36.24)\end{array}$ & $\begin{array}{l}35.3(30.96- \\
39.65)\end{array}$ & $\begin{array}{l}34.11(32.76- \\
35.47)\end{array}$ & $\begin{array}{l}35.69(34.35- \\
37.03)\end{array}$ & $\begin{array}{l}24.97(15.13- \\
34.82)\end{array}$ & $\begin{array}{l}23.33(15.36- \\
31.31)\end{array}$ & $\begin{array}{l}27.75(24.48- \\
31.02)\end{array}$ & $\begin{array}{l}29.52(25.06- \\
33.97)\end{array}$ \\
\hline $35-44 \mathrm{Yr}$ & $11.56(9.3-13.82)$ & $\begin{array}{l}11.33(8.64- \\
14.02)\end{array}$ & $\begin{array}{l}14.06(12.94- \\
15.18)\end{array}$ & $\begin{array}{l}15.05(13.86- \\
16.25)\end{array}$ & $4.11(0.32-7.91)$ & $4.9(0-10.58)$ & $4.57(3.05-6.09)$ & $5.12(3.18-7.07)$ \\
\hline $45-54 \mathrm{Yr}$ & $6.65(4.64-8.65)$ & $5.88(3.25-8.5)$ & $8.26(7.51-9.01)$ & $7.31(6.55-8.08)$ & $2.14(0-4.66)$ & $2.51(0-7.5)$ & $2.00(0.77-3.22)$ & $1.66(0.48-2.84)$ \\
\hline$\geq 55 \mathrm{Yr}$ & $5.98(3.89-8.07)$ & $5.18(3.14-7.23)$ & $8.93(8.2-9.66)$ & $7.58(6.85-8.32)$ & $1.36(0-3.95)$ & $0(0-0)$ & $0.79(0.12-1.46)$ & $0.68(0-1.51)$ \\
\hline \multicolumn{9}{|l|}{$\operatorname{Sex}^{\mathrm{a}}$} \\
\hline Male & $\begin{array}{l}37.03(33.77- \\
40.28)\end{array}$ & $\begin{array}{l}39.96(35.76- \\
44.15)\end{array}$ & $\begin{array}{l}59.31(58.2- \\
60.42)\end{array}$ & $\begin{array}{l}58.71(57.56- \\
59.87)\end{array}$ & $\begin{array}{l}28.16(19.05- \\
37.27)\end{array}$ & $\begin{array}{l}34.56(25.21- \\
43.91)\end{array}$ & $\begin{array}{l}66.97(64.17- \\
69.77)\end{array}$ & $\begin{array}{l}64.78(60.79- \\
68.76)\end{array}$ \\
\hline Female & $\begin{array}{l}62.97(59.72- \\
66.23)\end{array}$ & $\begin{array}{l}60.04(55.85- \\
64.24)\end{array}$ & $\begin{array}{l}40.69(39.58- \\
41.8)\end{array}$ & $\begin{array}{l}41.29(40.13- \\
42.44)\end{array}$ & $\begin{array}{l}71.84(62.73- \\
80.95)\end{array}$ & $\begin{array}{l}65.44 \text { (56.09- } \\
74.79)\end{array}$ & $\begin{array}{l}33.03(30.23- \\
35.83)\end{array}$ & $\begin{array}{l}35.22(31.24- \\
39.21)\end{array}$ \\
\hline \multicolumn{9}{|l|}{ Race } \\
\hline $\begin{array}{l}\text { White, non- } \\
\text { Hispanic }\end{array}$ & $\begin{array}{l}59.67(55.81- \\
63.53)\end{array}$ & $\begin{array}{l}55.94(51.96- \\
59.91)\end{array}$ & $\begin{array}{l}62.24(60.67- \\
63.82)\end{array}$ & $\begin{array}{l}60.73(59.08- \\
62.38)\end{array}$ & $\begin{array}{l}58.22(48.65- \\
67.8)\end{array}$ & $\begin{array}{l}55.02(42.85- \\
67.19)\end{array}$ & $\begin{array}{l}55.47(51.38- \\
59.57)\end{array}$ & $\begin{array}{l}48.90(44.07- \\
53.74)\end{array}$ \\
\hline $\begin{array}{l}\text { Black, non- } \\
\text { Hispanic }\end{array}$ & $\begin{array}{l}13.47(10.89- \\
16.05)\end{array}$ & $\begin{array}{l}12.31(10.03- \\
14.59)\end{array}$ & $\begin{array}{l}10.21(9.27- \\
11.16)\end{array}$ & $\begin{array}{l}11.31(10.34- \\
12.29)\end{array}$ & $\begin{array}{l}11.21(5.49- \\
16.94)\end{array}$ & $12.98(5.7-20.26)$ & $\begin{array}{l}11.17(8.21- \\
14.13)\end{array}$ & $\begin{array}{l}12.63(8.79- \\
16.47)\end{array}$ \\
\hline $\begin{array}{l}\text { Other, non- } \\
\text { Hispanic }\end{array}$ & $7.7(5.82-9.57)$ & $7.88(5.66-10.09)$ & $9.84(8.75-10.93)$ & $9.98(9.01-10.96)$ & $6.81(2.93-10.69)$ & $9.14(4.62-13.66)$ & $\begin{array}{l}11.19(8.18- \\
14.19)\end{array}$ & $\begin{array}{l}15.60(11.83- \\
19.37)\end{array}$ \\
\hline Hispanic & $\begin{array}{l}19.16(16.16- \\
22.16)\end{array}$ & $\begin{array}{l}23.88(20.48- \\
27.28)\end{array}$ & $\begin{array}{l}17.7(16.45- \\
18.95)\end{array}$ & $\begin{array}{l}17.98(16.67- \\
19.29)\end{array}$ & $\begin{array}{l}23.75(14.76- \\
32.73)\end{array}$ & $\begin{array}{l}22.86(12.13- \\
33.59)\end{array}$ & $\begin{array}{l}22.17(18.8- \\
25.53)\end{array}$ & $\begin{array}{l}22.86(18.7- \\
27.02)\end{array}$ \\
\hline \multicolumn{9}{|l|}{ Education Level } \\
\hline No College & $\begin{array}{l}32.01(28.77- \\
35.24)\end{array}$ & $\begin{array}{l}34.29(30.33- \\
38.25)\end{array}$ & $\begin{array}{l}30.21(28.94- \\
31.48)\end{array}$ & $30(28.52-31.47)$ & $\begin{array}{l}36.28(26.49- \\
46.06)\end{array}$ & $\begin{array}{l}46.14(34.05- \\
58.23)\end{array}$ & $\begin{array}{l}36.3(33.19- \\
39.41)\end{array}$ & $\begin{array}{l}37.22(32.72- \\
41.73)\end{array}$ \\
\hline Some College & $\begin{array}{l}67.99(64.76- \\
71.23)\end{array}$ & $\begin{array}{l}65.71(61.75- \\
69.67)\end{array}$ & $\begin{array}{l}69.79(68.52- \\
71.06)\end{array}$ & $70(68.53-71.48)$ & $\begin{array}{l}63.72(53.94- \\
73.51)\end{array}$ & $\begin{array}{l}53.86(41.77- \\
65.95)\end{array}$ & $\begin{array}{l}63.7(60.59- \\
66.81)\end{array}$ & $\begin{array}{l}62.78(58.27- \\
67.28)\end{array}$ \\
\hline \multicolumn{9}{|l|}{ Marital Status } \\
\hline Married & - & $\begin{array}{l}17.03(13.97- \\
20.1)\end{array}$ & - & $\begin{array}{l}31.89(30.65- \\
33.13)\end{array}$ & - & $\begin{array}{l}10.57(4.21- \\
16.93)\end{array}$ & - & $\begin{array}{l}15.36(11.58- \\
19.15)\end{array}$ \\
\hline Not Married $t$ & - & $\begin{array}{l}82.97(79.9- \\
86.03)\end{array}$ & - & $\begin{array}{l}68.11(66.87- \\
69.35)\end{array}$ & - & $\begin{array}{l}89.43(83.07- \\
95.79)\end{array}$ & - & $\begin{array}{l}84.64(80.85- \\
88.42)\end{array}$ \\
\hline \multicolumn{9}{|c|}{ Annual Household Income } \\
\hline$<\$ 25,000$ & $\begin{array}{l}47.09(43.49- \\
50.7)\end{array}$ & $\begin{array}{l}49.27(45.25- \\
53.3)\end{array}$ & $\begin{array}{l}35.23(33.81- \\
36.66)\end{array}$ & $\begin{array}{l}34.33(32.79- \\
35.87)\end{array}$ & $\begin{array}{l}58.96(48.67- \\
69.26)\end{array}$ & $\begin{array}{l}54.24(44.53- \\
63.94)\end{array}$ & $\begin{array}{l}49.15(45.7- \\
52.59)\end{array}$ & $\begin{array}{l}46.16(41.72- \\
50.6)\end{array}$ \\
\hline$\$ 25,000-49,999$ & $\begin{array}{l}24.01(21.51- \\
26.52)\end{array}$ & $\begin{array}{l}20.36(17.08- \\
23.64)\end{array}$ & $23.03(22-24.05)$ & $\begin{array}{l}21.91(20.8- \\
23.02)\end{array}$ & $\begin{array}{l}24.74(15.88- \\
33.59)\end{array}$ & $\begin{array}{l}25.35(17.61- \\
33.1)\end{array}$ & $\begin{array}{l}22.8(19.69- \\
25.92)\end{array}$ & $\begin{array}{l}18.08(14.12- \\
22.03)\end{array}$ \\
\hline$\$ 50,000-99,000$ & $\begin{array}{l}18.82(15.79- \\
21.85)\end{array}$ & $\begin{array}{l}20.36(17.08- \\
23.64)\end{array}$ & $\begin{array}{l}23.35(22.23- \\
24.47)\end{array}$ & $\begin{array}{l}24.07(22.78- \\
25.36)\end{array}$ & $\begin{array}{l}11.96(4.27- \\
19.64)\end{array}$ & $\begin{array}{l}12.72(6.32- \\
19.13)\end{array}$ & $\begin{array}{l}16.08(13.24- \\
18.93)\end{array}$ & $\begin{array}{l}19.7(16.03- \\
23.37)\end{array}$ \\
\hline$\geq \$ 100,000$ & $10.07(7.9-12.23)$ & $9.99(7.57-12.42)$ & $\begin{array}{l}18.39(17.13- \\
19.65)\end{array}$ & $\begin{array}{l}19.69(18.25- \\
21.13)\end{array}$ & $4.34(0.69-8)$ & $7.69(0.69-14.68)$ & $\begin{array}{l}11.97(9.42- \\
14.51)\end{array}$ & $\begin{array}{l}16.06(11.85- \\
20.28)\end{array}$ \\
\hline \multicolumn{9}{|l|}{ Health insurance } \\
\hline No & $\begin{array}{l}21.97(19.24- \\
24.71)\end{array}$ & $\begin{array}{l}24.08(20.59- \\
27.57)\end{array}$ & $\begin{array}{l}18.65(17.65- \\
19.66)\end{array}$ & $\begin{array}{l}16.78(15.55- \\
18.02)\end{array}$ & $\begin{array}{l}26.48(19.1- \\
33.86)\end{array}$ & $\begin{array}{l}29.87(20.3- \\
39.45)\end{array}$ & $\begin{array}{l}23.5(19.53- \\
27.48)\end{array}$ & $\begin{array}{l}21.87(17.84- \\
25.91)\end{array}$ \\
\hline Yes & $\begin{array}{l}78.03 \text { (75.29- } \\
80.76)\end{array}$ & $\begin{array}{l}75.92(72.43- \\
79.41)\end{array}$ & $\begin{array}{l}81.35(80.34- \\
82.35)\end{array}$ & $\begin{array}{l}83.22(81.98- \\
84.45)\end{array}$ & $\begin{array}{l}73.52(66.14- \\
80.9)\end{array}$ & $\begin{array}{l}70.13(60.55- \\
79.7)\end{array}$ & $\begin{array}{l}76.5(72.52- \\
80.47)\end{array}$ & $\begin{array}{l}78.13(74.09- \\
82.16)\end{array}$ \\
\hline
\end{tabular}

* African-American adults were oversampled and percentages were weighted to represent the U.S. adult populations. Data are shown as percent (95\% Cl) tIncluding widowed, divorced, separated, or never married

Dash (-) indicates questions were not asked in Wave 1

a $p<0.0001$ for comparison sexual identity by gender within wave 1 and within wave 2 for those with ever use and for those with current use

smoke only hookah at Wave 2; 4.0\% transitioned to use of other tobacco products in addition to hookah use at Wave 2; 11.0\% discontinued hookah use and switched to other tobacco products at Wave 2; and 46.2\% quit hookah use (and used no other tobacco products) at Wave 2. Among heterosexual adults, the transition pattern was 


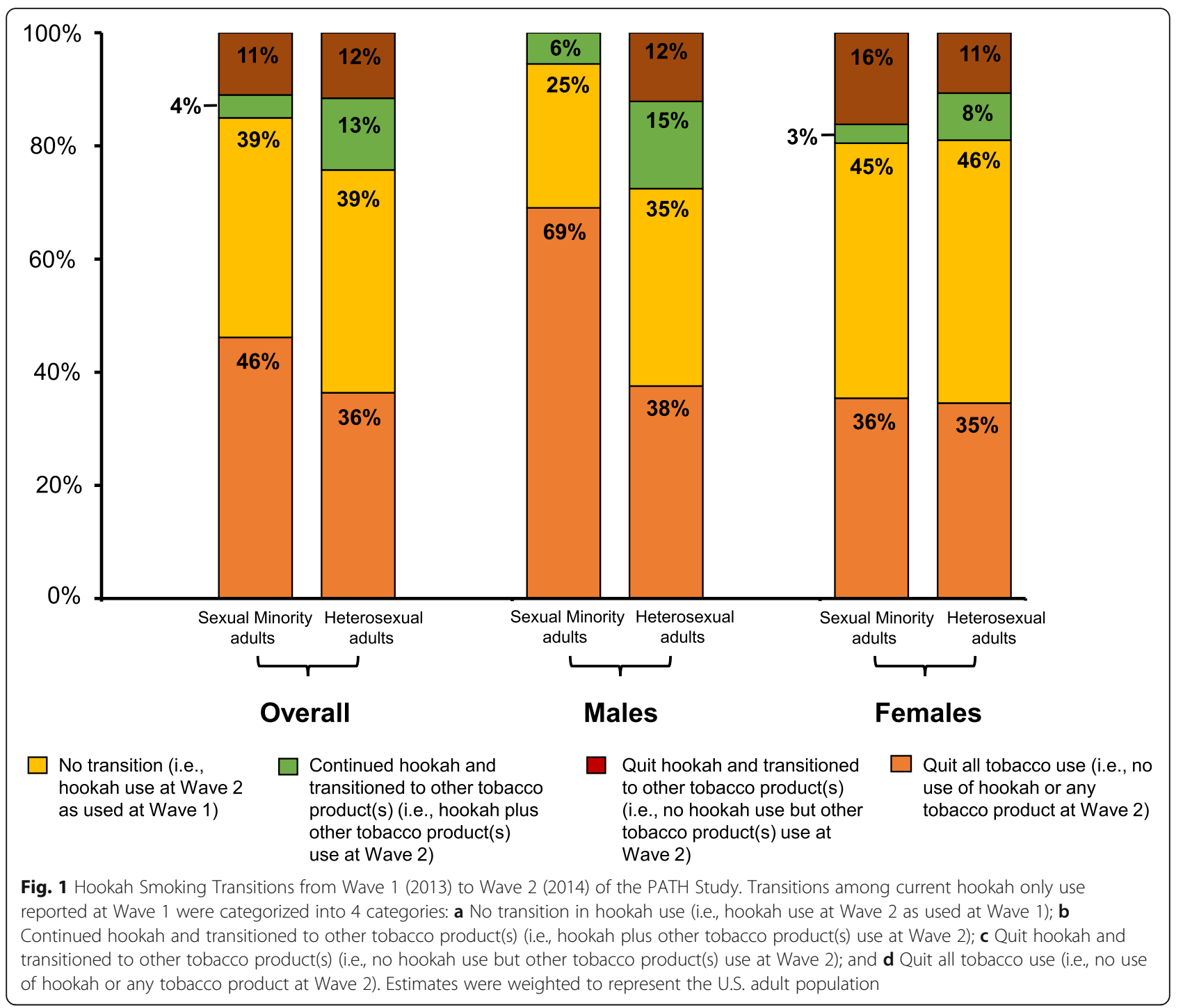

not different from SM adults: among current hookah-only smokers at Wave 1, 39.4\% continued to smoke only hookah at Wave 2; $12.7 \%$ added other tobacco products use in addition to hookah use in Wave 2; 11.6\% discontinued hookah use but used other tobacco products at Wave 2; and 36.4\% quit hookah use (and used no other tobacco products) at Wave 2 .

Compared with heterosexual men, SM men who reported current hookah use at Wave 1 , a greater percentage reported quitting completely at Wave 2 (38\% vs. 69\%, $p<$ 0.05). No significant differences were observed among heterosexual vs. SM women ( $35 \%$ vs. $36 \%, p=n s)$. Compared to heterosexual adults, more than half of SM adults age 25-34 years reported quitting hookah use at Wave 2 (43\% vs. $63 \%, P<0.05$ ). Among the younger population (i.e., $18-$ 24 years), $41 \%$ of SM adults, compared to $36 \%$ heterosexual adults, reported quitting while $47 \%$ reported no transition in hookah use at Wave 2, compared to $42 \%$ among heterosexuals.

Results of the multinomial model of the four transition categories showed a gender main effect (Supplemental Table 2) with Wave 1 female hookah-only users less likely to discontinue all hookah and tobacco use by Wave 2 or to take up use of other tobacco products; that is, females were more likely to remain consistent in their hookah-only use. Unfortunately, limited data coverage did not allow the inclusion of interaction effects in the multivariable model; thus, the multivariate analysis could not confirm all the comparisons reported in the previous paragraphs.

\section{Co-use of tobacco, alternative tobacco product and electronic nicotine products}

As shown in Fig. 2 and Fig. 3, cigarettes were the most common tobacco product used in combination with 


\section{Sexual Minority Hookah Smokers}

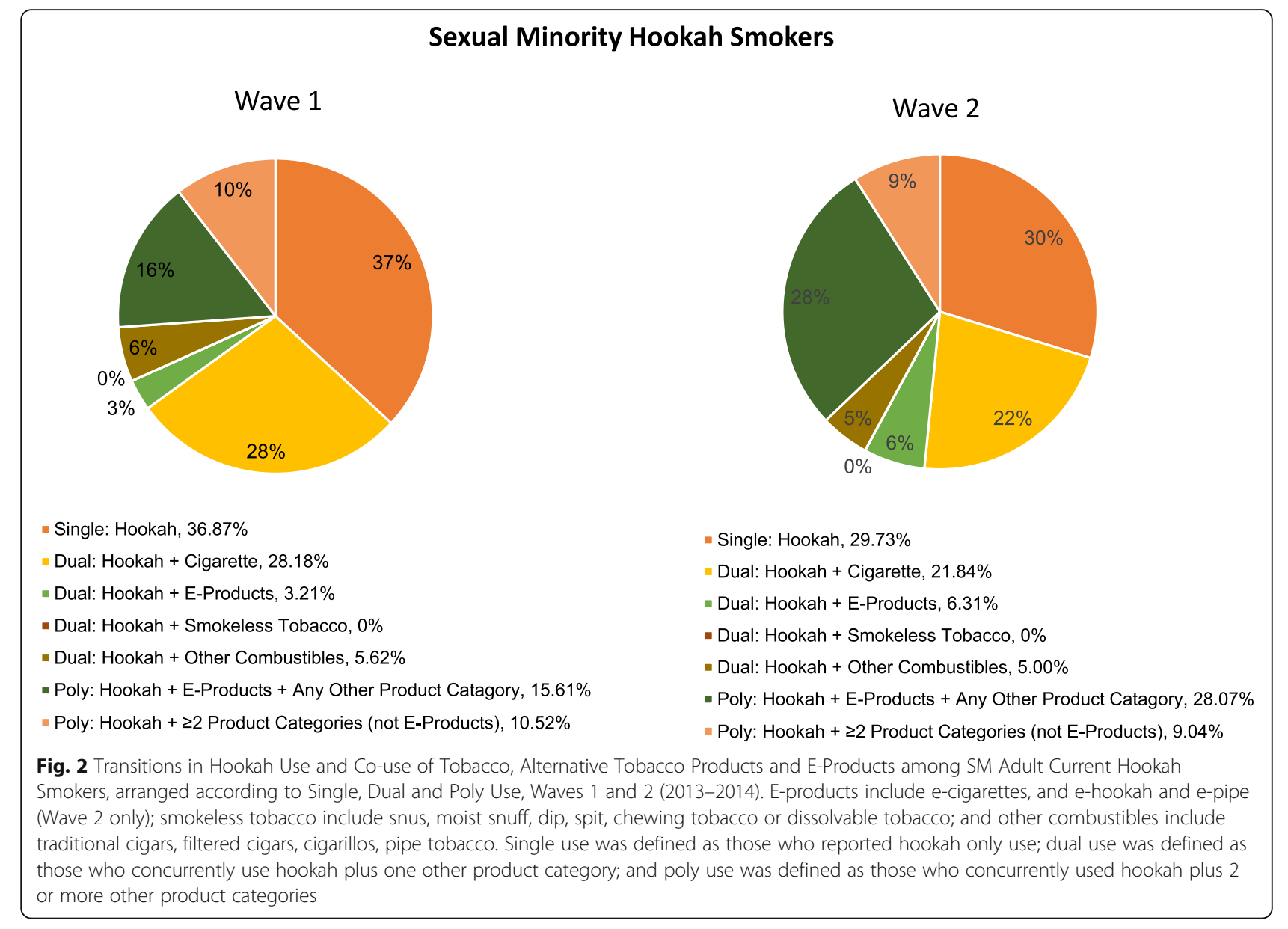

hookah among Wave 1 and Wave 2 SM and heterosexual adult current hookah users. Among Wave 1 SM hookah users, $36.87 \%$ reported single hookah use, $37.00 \%$ reported hookah dual use and $25.04 \%$ poly hookah use. While single and dual hookah use decreased among SM adults in Wave 2 (29.73 and 33.16\%, respectively), poly use increased to $35.08 \%$. Among Wave 1 heterosexual adult hookah users, $45.86 \%$ reported single hookah use, $32.21 \%$ reported hookah dual use and $20.76 \%$ poly hookah use. In Wave 2, single hookah use decreased to $40.19 \%$ but both dual and poly use increased among heterosexuals in Wave 2 (36.09 and $23.00 \%$, respectively). Dual hookah plus e-products use increased similarly among SM and heterosexual adults from Wave 1 to Wave 2 (increased by 97 and 99\%, respectively). A higher percentage of SM adults reported poly hookah use at Wave 2 compared with heterosexual adults (increased by $40 \%$ vs. $11 \%$, respectively). While hookah plus e-products use (with or without other tobacco product(s)) in Wave 2 increased significantly among SM adults (increased by 65 and 83\%, respectively), both hookah use and hookah plus other tobacco use decreased similarly among SM and heterosexual adults (Fig. 4).
An additional perspective of Wave 2 initiation of multiproduct use by Wave 1 current hookah users is provided by the multinomial logistic results of selected transition patterns (Supplemental Table 3); the model included sexual minority status as a predictor, as well as gender, age, race/ethnicity, and health insurance status. Four transition patterns were considered: consistent hookah and other product use across the two waves, initiation of e-products with continued hookah use, other change in multiproduct use, and cessation of hookah use. Few differences in transitions were distinguishable in terms of sociodemographic characteristics. In this multivariable model, nonHispanic Blacks were more likely $(\mathrm{OR}=1.22)$ than were Hispanics to initiate e-products over consistent product use. A significant age by sexual minority status interaction was seen for specifically cessation of hookah use as compared to consistent product use: older sexual minorities were most likely to cease hookah use than maintain a consistent multi-product use pattern $(\mathrm{OR}=5.93)$, calculated from coefficients shown in Supplemental Table 3. This pattern of cessation of hookah use is consistent with the simpler comparative results described in the previous section for the subsample of hookah only users. 


\section{Heterosexual Hookah Smokers}

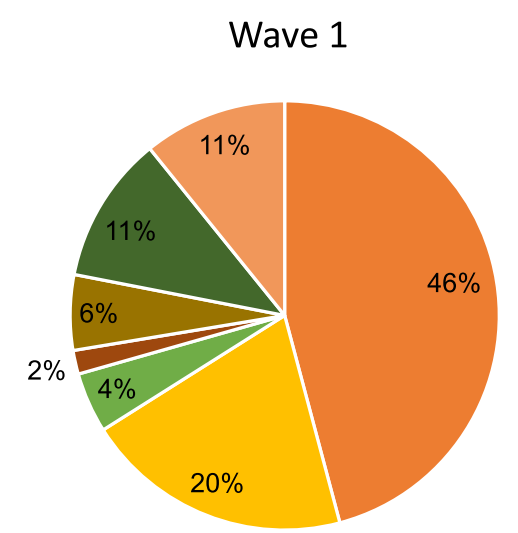

- Single: Hookah, $45.86 \%$

Dual: Hookah + Cigarette, $20.19 \%$

- Dual: Hookah + E-Products, $4.55 \%$

- Dual: Hookah + Smokeless Tobacco, 1.79\%

- Dual: Hookah + Other Combustibles, $5.68 \%$

- Poly: Hookah + E-Products + Any Other Product Catagory, 11.12\%

- Poly: Hookah $+\geq 2$ Product Categories (not E-Products), $10.82 \%$
Wave 2

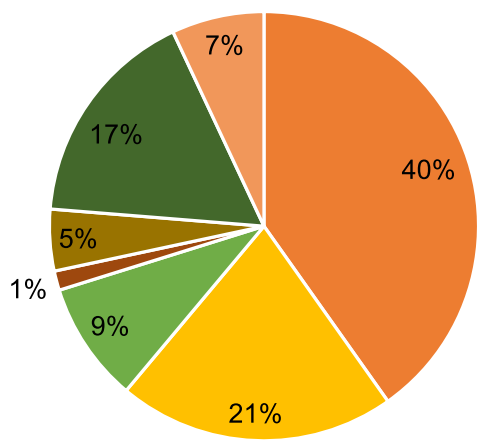

- Single: Hookah, 40.19\%

- Dual: Hookah + Cigarette, $20.91 \%$

- Dual: Hookah + E-Products, 9.08\%

- Dual: Hookah + Smokeless Tobacco, 1.46\%

- Dual: Hookah + Other Combustibles, 4.64\%

- Poly: Hookah + E-Products + Any Other Product Catagory, 16.75\%

- Poly: Hookah $+\geq 2$ Product Categories (not E-Products), $6.97 \%$

Fig. 3 Transitions in Hookah Use and Co-use of Tobacco, Alternative Tobacco Products and E-Products among Heterosexual Adult Current Hookah Smokers, arranged according to Single, Dual and Poly Use, Waves 1 and 2 (2013-2014). E-products include e-cigarettes, e-hookah and epipe (Wave 2 only); smokeless tobacco include snus, moist snuff, dip, spit, chewing tobacco or dissolvable tobacco; and other combustibles include traditional cigars, filtered cigars, cigarillos, pipe tobacco. Single use was defined as those who reported hookah only use; dual use was defined as those who concurrently use hookah plus one other product category; and poly use was defined as those who concurrently used hookah plus 2 or more other product categories

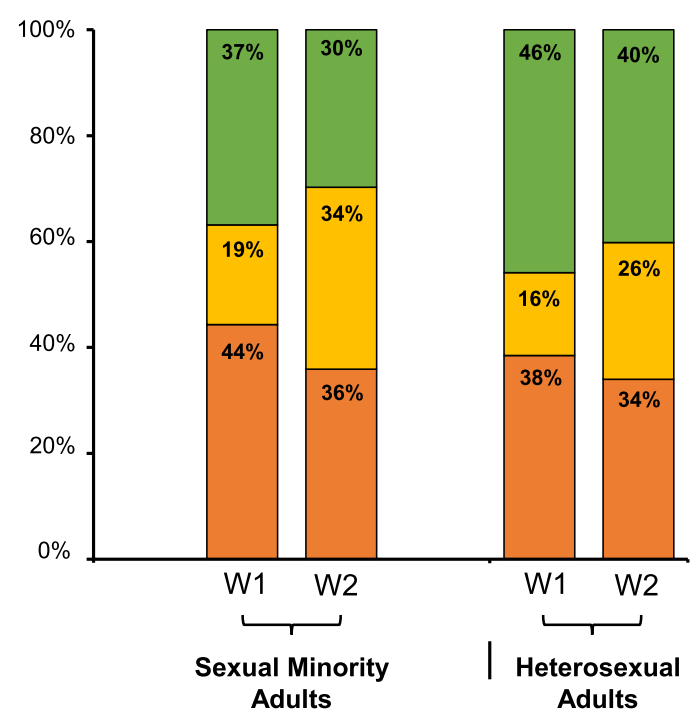

\section{Hookah only}

Hookah + E-Products (with or without other tobacco products)

\section{Hookah + Alternative Tobacco} Products (no e-products)

Fig. 4 Transitions in Hookah Use and Co-use of E-Products and Alternative Tobacco Products among SM vs. Heterosexual Adult Current Hookah Smokers, Waves 1 and 2 (2013-2014). E-products include e-cigarettes, and e-hookah and e-pipe (Wave 2 only); and alternative tobacco products include cigarettes, smokeless tobacco (i.e., snus, moist snuff, dip, spit, chewing tobacco or dissolvable tobacco) and other combustibles (i.e., traditional cigars, filtered cigars, cigarillos, pipe tobacco) 


\section{Discussion}

Using nationally representative data, we sought to characterize transitions between hookah smoking and use of other tobacco products among SM adults versus their heterosexual counterparts. This study provides two novel insights into these transitions. First, our results demonstrate higher rates of ever and current hookah use among SM adults compared to their heterosexual counterparts. Second, while $46 \%$ of SM adults reported quitting hookah smoking at Wave 2, among current hookah users, hookah plus e-product (with or without other tobacco product(s)) use markedly increased at Wave 2 among SM adults (Wave 1: 19\%; Wave 2: 34\%), compared to increases among heterosexual individuals (Wave 1: 16\%; Wave 2: 26\%). It is noteworthy that among SM adult current hookah smokers, dual hookah plus e-product use (without other tobacco product(s)) increased by 97\% at Wave 2 (Wave 1: 3\%; Wave 2: 6\%), with comparable trends among heterosexual individuals (Wave 1: 5\%; Wave 2: 9\%).

While the investigation into the cause of the recent epidemic of vaping-induced deaths and illness is still ongoing [21, 22], our findings highlight vital trends regarding the rapid uptake of vaping-using various e-products such as e-cigarettes, e-hookahs and e-pipes-among SM hookah smokers. In a two-year period, our nationally representative findings show that hookah plus e-product use (with or without other tobacco product(s)) increased by $83 \%$ among SM adults, compared to $65 \%$ among heterosexual individuals. In light of these findings, and because there is limited evidence for interventions to address common misperceptions on potential hookah harms $[23,24]$, our study emphasize the need for strong efforts to increase awareness of the harmful effects of hookah as well as vaping, targeted towards sexual minority populations. Our findings also illustrate the importance of feasible and effective health education programing and communication efforts, specifically tailored to SM communities. For example, special programing that could potentially prevent the onset or continued use of hookah and vaping products and assist with cessation programs aimed at reaching SM populations. Indeed, evidence suggests that few anti-tobacco campaigns have been designed specifically to reach sexual minority populations [25].

Use of alternative tobacco products such as hookah has risen abruptly in the past decade $[9,26]$. Few studies have examined hookah use among SM populations. Prior analysis of PATH data from Wave 1 found that SM individuals had higher odds of hookah use compared to heterosexual individuals [10]. Similarly, nationally representative data from Legacy's Young Adult Cohort Study show that ever hookah use was significantly higher among SM respondents compared with those who identified as heterosexuals [27]. Our analyses confirm these findings by showing that over a two-year period, SM adults continue to have significantly higher rates of hookah use compared with heterosexual adults. Furthermore, our analysis extends these findings by demonstrating that a larger percentage of SM adult, specifically male hookah smokers, compared to heterosexuals, reported quitting hookah smoking at Wave 2. While is it unknown whether these individuals may return to use hookah, future analysis of additional waves of the PATH study may provide further insight into longer-term patterns of hookah use within SM populations.

There is growing concern that hookah smoking may function as a gateway to other tobacco products and harmful substances. Recent prospective analysis from the PATH study 2013-2015 indicate that hookah use is independently associated with subsequent smoking in the year ahead [28]. This finding is consistent with other studies that demonstrate hookah use is associated with more than double the odds of subsequent initiation of cigarette smoking [29]. Our analyses demonstrate that a large majority of SM current hookah smokers $(63 \%$ in Wave 1 and $70 \%$ in Wave 2) reported using hookah plus other tobacco products, with cigarettes being the most common tobacco product used in combination with hookah. While multiple factors may explain our findings, flavored tobacco products have been previously demonstrated to serve as starter products to regular tobacco use [30]. Indeed, sexual minority status has been shown to be associated with use of flavored tobacco products $[31,32]$, and evidence show that the tobacco industry has selectively targeted the marketing of products to sexual minority individuals $[12,13,15,17]$. In addition to tobacco and menthol flavors, hookah tobacco come in fruit, candy, and alcohol flavors and while the 2009 Family Smoking Prevention and Tobacco Control Act banned characterizing flavors other than menthol in cigarettes, this ban does not extend to hookah [33]. Our findings build upon previous work highlighting the need for robust regulation to reduce flavored tobacco appeal specifically among SM communities.

There are several limitations to this study. Respondents' smoking status was not biochemically verified. Although this study focused exclusively on SM adults, it is important to note gender differences within SM and heterosexual samples when addressing transitions. Combining sexual minority subgroups (i.e., lesbian women, bisexual men) may mask unique differences with regards to hookah use prevalence and transitions, and may obscure subgroup specific health needs. Because the PATH questionnaire's 'something else' category encompasses a highly heterogeneous group that may not necessarily represent the definition of "sexual minority" (i.e., genderqueer people), future research is needed to include 
specific questions to identify gender diverse individuals and better understand hookah tobacco trends among gender minorities as well as sexual minorities [34]. PATH Study data were self-reported and therefore responses may underrepresent the SM community because of the related-stigma surrounding sexual orientation. Further exploration is needed with longitudinal models that can accommodate the complex survey weights as well as capture behavior change and time-dependent covariates.

\section{Conclusions}

This study is one of the first to characterize quitting as well as transitions between hookah smoking and use of other tobacco products among SM adult hookah smokers using a nationally representative sample in the United States. In addition to higher rates of hookah use among SM adults, higher percentages of SM adults transitioned to hookah plus e-product use between 2013 and 2015 compared to their non-minority peers. Considering our findings in light of the study limitations and the context of the limited literature, future work should aim to further examine mechanisms that drive the higher rates of hookah use among SM individuals and how these drivers may differ by unique SM subgroups (i.e., socialization/affiliation versus stress processes may differ by subgroup). Finally, information regarding the harmful effects of hookah use should be tailored to reach diverse sexual minority communities.

\section{Supplementary Information}

The online version contains supplementary material available at https://doi. org/10.1186/s12889-021-10389-5.

Additional file 1: Supplemental Table 1. Sociodemographic Predictors of Ever and Current Hookah Use Waves 1 and 2 (2013-2014). Supplemental Table 2. Sociodemographic Predictors for Wave 1 Hookah-Only Users Transition Patterns. Supplemental Table 3. Sociodemographic Predictors of Transition Patterns Wave 1 to Wave 2 (Including Uptake of E-Products)

\section{Abbreviations}

PATH: Population Assessment of Tobacco and Health; SM: Sexual minority

\section{Acknowledgments}

Not applicable.

\section{Authors' contributions}

$\mathrm{MRH}$ and LS conceptualized and designed the study. MRH and IWH drafted the initial manuscript. JT and USW carried out the analyses, reviewed and revised the manuscript. MRH and MLB performed quality assurance and control of the analyses. Others, including LCB and LS reviewed and approved the initial proposed analyses, reviewed and revised the manuscript. The authors read and approved the final manuscript

\section{Availability of data and materials}

The datasets generated and/or analysed during the current study are available from the Population Assessment of Tobacco and Health Study Public-Use Files, https://www.icpsr.umich.edu/icpsrweb/NAHDAP/studies/36498

\section{Ethics approval and consent to participate}

Ethics approval was obtained from the University of California, Los Angeles Institutional Review Board in order to access and analyze the study data.

\section{Consent for publication}

Not applicable.

\section{Competing interests}

The authors declare that they have no competing interests.

\section{Author details}

${ }^{1}$ School of Nursing, University of California, Los Angeles, 700 Tiverton Ave, 4-254 Factor Building, Los Angeles, CA 90095, USA. ²Department of Social Welfare, Luskin School of Public Affairs, University of California, Los Angeles, Los Angeles, CA, USA.

Received: 8 April 2020 Accepted: 4 February 2021

Published online: 05 March 2021

\section{References}

1. Maziak W, Taleb ZB, Bahelah R, Islam F, Jaber R, Auf R, et al. The global epidemiology of waterpipe smoking. Tob Control. 2015;24(Suppl 1):i3-i12.

2. Bhatnagar A, Maziak W, Eissenberg T, Ward KD, Thurston G, King BA, et al. Water Pipe (Hookah) Smoking and Cardiovascular Disease Risk: A Scientific Statement From the American Heart Association. Circulation. 2019: Cir0000000000000671.

3. Rezk-Hanna M, Benowitz NL. Cardiovascular effects of hookah smoking: potential implications for cardiovascular risk. Nicotine Tobacco Res 20185 [Epub ahead of print].

4. Rezk-Hanna M, Macabasco-O'Connell A, Woo M. Hookah smoking among young adults in southern California. Nurs Res. 2014;63(4):300-6.

5. Delahanty J, Ganz O, Hoffman L, Guillory J, Crankshaw E, Farrelly M. Tobacco use among lesbian, gay, bisexual and transgender young adults varies by sexual and gender identity. Drug Alcohol Depend. 2019;201:161-70.

6. Wang TW, Asman K, Gentzke AS, Cullen KA, Holder-Hayes E, Reyes-Guzman C, et al. Tobacco product use among adults - United States, 2017. MMWR Morb Mortal Wkly Rep. 2018;67(44):1225-32.

7. Johnson SE, Holder-Hayes E, Tessman GK, King BA, Alexander T, Zhao X. Tobacco product use among sexual minority adults: findings from the 20122013 National Adult Tobacco Survey. Am J Prev Med. 2016;50(4):e91-e100.

8. Bhatnagar A, Whitsel LP, Blaha MJ, Huffman MD, Krishan-Sarin S, Maa J, et al. New and emerging tobacco products and the nicotine endgame: the role of robust regulation and comprehensive tobacco control and prevention: a presidential advisory from the American Heart Association. Circulation. 2019; 139(19):e937-e58.

9. Kasza KA, Ambrose BK, Conway KP, Borek N, Taylor K, Goniewicz ML, et al. Tobacco-product use by adults and youths in the United States in 2013 and 2014. N Engl J Med. 2017;376(4):342-53.

10. Wheldon CW, Kaufman AR, Kasza KA, Moser RP. Tobacco use among adults by sexual orientation: findings from the population assessment of tobacco and health study. LGBT Health. 2018;5(1):33-44.

11. Blosnich J, Lee JG, Horn K. A systematic review of the aetiology of tobacco disparities for sexual minorities. Tob Control. 2013;22(2):66-73.

12. Stevens $P$, Carlson LM, Hinman JM. An analysis of tobacco industry marketing to lesbian, gay, bisexual, and transgender (LGBT) populations: strategies for mainstream tobacco control and prevention. Health Promot Pract. 2004;5(3 Suppl):129s-34s.

13. Soneji S, Knutzen KE, Tan ASL, Moran MB, Yang J, Sargent J, et al. Online tobacco marketing among US adolescent sexual, gender, racial, and ethnic minorities. Addict Behav. 2019;95:189-96.

14. Ryan H, Wortley PM, Easton A, Pederson L, Greenwood G. Smoking among lesbians, gays, and bisexuals: a review of the literature. Am J Prev Med. 2001:21(2):142-9.

15. Emory K, Buchting FO, Trinidad DR, Vera L, Emery SL. Lesbian, gay, bisexual, and transgender (LGBT) view it differently than non-LGBT: exposure to tobacco-related couponing, E-cigarette advertisements, and anti-tobacco
Funding

Not applicable. 
messages on social and traditional media. Nicotine Tobacco Res. 2019;21(4): 513-22.

16. Tan ASL, Hanby EP, Sanders-Jackson A, Lee S, Viswanath K, Potter J. Inequities in tobacco advertising exposure among young adult sexual, racial and ethnic minorities: examining intersectionality of sexual orientation with race and ethnicity. Tob Control. 2021;30(1):84-93.

17. Dilley JA, Spigner C, Boysun MJ, Dent CW, Pizacani BA. Does tobacco industry marketing excessively impact lesbian, gay and bisexual communities? Tob Control. 2008;17(6):385-90.

18. Kasza KA, Borek N, Conway KP, Goniewicz ML, Stanton CA, Sharma E, et al. Transitions in Tobacco Product Use by U.S. Adults between 2013(-)2014 and 2014(-)2015: Findings from the PATH Study Wave 1 and Wave 2. Int J Environ Res Public Health. 2018;15:11.

19. Hyland A, Ambrose BK, Conway KP, Borek N, Lambert E, Carusi C, et al. Design and methods of the population assessment of tobacco and health (PATH) study. Tob Control. 2017;26(4):371-8.

20. National Addiction and HIV Data Archive Program (NAHDAP). Population Assessment of Tobacco and Health (PATH) Study Series. https://www.icpsr. umich.edu/icpsrweb/NAHDAP/series/606. Accessed 17 March 2020.

21. Layden JE, Ghinai I, Pray I, Kimball A, Layer M, Tenforde M, et al. Pulmonary illness related to E-cigarette use in Illinois and Wisconsin - preliminary report. N Engl J Med. 2020;382(10):903-16.

22. Marsden L, Michalicek ZD, Christensen ED. More on the pathology of Vaping-associated lung injury. N Engl J Med. 2020;382(4):387-8.

23. Jawad M, Jawad S, Waziry RK, Ballout RA, Akl EA. Interventions for waterpipe tobacco smoking prevention and cessation: a systematic review. Sci Rep. 2016;6:25872.

24. Maziak W, Jawad M, Jawad S, Ward KD, Eissenberg T, Asfar T. Interventions for waterpipe smoking cessation. Cochrane Database Syst Rev. 2015;(7): Cd005549.

25. Matthews AK, Balsam K, Hotton A, Kuhns L, Li CC, Bowen DJ. Awareness of media-based antitobacco messages among a community sample of LGBT individuals. Health Promot Pract. 2014;15(6):857-66.

26. Robinson J, Wang B, Jackson K, Donaldson E, Ryant C. Characteristics of hookah tobacco smoking sessions and correlates of use frequency among US adults: findings from wave 1 of the population assessment of tobacco and health (PATH) study. Nicotine Tobacco Res. 2018;20(6):731-40.

27. Rath JM, Villanti AC, Rubenstein RA, Vallone DM. Tobacco use by sexual identity among young adults in the United States. Nicotine Tobacco Res. 2013;15(11):1822-31.

28. Watkins SL, Glantz SA, Chaffee BW. Association of Noncigarette Tobacco Product use with Future Cigarette Smoking among Youth in the population assessment of tobacco and health (PATH) study, 2013-2015. JAMA Pediatr. 2018;172(2):181-7.

29. Al Oweini $D$, Jawad M, Akl EA. The association of waterpipe tobacco smoking with later initiation of cigarette smoking: a systematic review and meta-analysis exploring the gateway theory. Tob Control. 2019.

30. Villanti AC, Johnson AL, Ambrose BK, Cummings KM, Stanton CA, Rose SW et al. Flavored tobacco product use in youth and adults: findings from the first wave of the PATH study (2013-2014). Am J Prev Med. 2017;53(2):13951

31. King BA, Dube SR, Tynan MA. Flavored cigar smoking among U.S. adults: findings from the 2009-2010 National Adult Tobacco Survey. Nicotine Tobacco Res. 2013;15(2):608-14.

32. Fallin A, Goodin AJ, King BA. Menthol cigarette smoking among lesbian, gay, bisexual, and transgender adults. Am J Prev Med. 2015;48(1):93-7.

33. US Department of Health and Human Services Food and Drug Administration. Deeming Tobacco Products To Be Subject to the Federal Food, Drug, and Cosmetic Act, as Amended by the Family Smoking Prevention and Tobacco Control Act. 21 CFR Parts 1100, 1140, and 1143. 2016. Vol. 81. Washington: Federal Register; 2016. May 10. p. 28974-9106.

34. Ferguson A, Gilmour M. Non-Monosex research publication in U.S.-based social work journals between 2008-2016. J Evid-Inform Soc Work. 2018;15(1): 23-37.

\section{Publisher's Note}

Springer Nature remains neutral with regard to jurisdictional claims in published maps and institutional affiliations.

\section{Ready to submit your research? Choose BMC and benefit from}

- fast, convenient online submission

- thorough peer review by experienced researchers in your field

- rapid publication on acceptance

- support for research data, including large and complex data types

- gold Open Access which fosters wider collaboration and increased citations

- maximum visibility for your research: over $100 \mathrm{M}$ website views per year

At BMC, research is always in progress.

Learn more biomedcentral.com/submissions 